OPEN ACCESS

Edited by:

M. Zachary Rosenthal,

Duke University, United States

Reviewed by:

Warren Mansell,

The University of Manchester,

United Kingdom

Santiago J. Ballaz,

Yachay Tech University, Ecuador

*Correspondence: Sari Goldstein Ferber

sari.goldstein@biu.ac.l

Specialty section: This article was submitted to

Psychopathology, a section of the journal

Frontiers in Psychiatry

Received: 14 March 2021

Accepted: 16 June 2021

Published: 29 July 2021

Citation:

Goldstein Ferber S, Shoval G Zalsman G, Mikulincer M and Weller A (2021) Between Action and Emotiona

Survival During the COVID-19 era: Sensorimotor Pathways as Control

Systems of Transdiagnostic

Anxiety-Related Intolerance to

Uncertainty.

Front. Psychiatry 12:680403. doi: 10.3389/fpsyt.2021.680403

\section{Between Action and Emotional Survival During the COVID-19 era: Sensorimotor Pathways as Control Systems of Transdiagnostic Anxiety-Related Intolerance to Uncertainty}

\author{
Sari Goldstein Ferber ${ }^{1 *}$, Gal Shoval2,3,4, Gil Zalsman ${ }^{2,3,5}$, Mario Mikulincer ${ }^{6}$ and \\ Aron Weller ${ }^{1}$
}

\begin{abstract}
1 Psychology Department and Gonda Brain Research Center, Bar-llan University, Ramat Gan, Israel, ${ }^{2}$ Geha Mental Health Center, Petah Tiqva, Israel, ${ }^{3}$ Sackler Faculty of Medicine, Tel Aviv University, Tel Aviv, Israel, ${ }^{4}$ Princeton Neuroscience Institute, Princeton University, Princeton, NJ, United States, ${ }^{5}$ Division of Molecular Imaging and Neuropathology, Department of Psychiatry, Columbia University and New York State Psychiatric Institute, New York, NY, United States, ${ }^{6}$ Interdisciplinary Center (IDC) Herzliya, Baruch Ivcher School of Psychology, Herzliya, Israel
\end{abstract}

Objectives: The COVID-19 pandemic and aligned social and physical distancing regulations increase the sense of uncertainty, intensifying the risk for psychopathology globally. Anxiety disorders are associated with intolerance to uncertainty. In this review we describe brain circuits and sensorimotor pathways involved in human reactions to uncertainty. We present the healthy mode of coping with uncertainty and discuss deviations from this mode.

Methods: Literature search of PubMed and Google Scholar.

Results: As manifestation of anxiety disorders includes peripheral reactions and negative cognitions, we suggest an integrative model of threat cognitions modulated by sensorimotor regions: "The Sensorimotor-Cognitive-Integration-Circuit." The model emphasizes autonomic nervous system coupling with the cortex, addressing peripheral anxious reactions to uncertainty, pathways connecting cortical regions and cost-reward evaluation circuits to sensorimotor regions, filtered by the amygdala and basal ganglia. Of special interest are the ascending and descending tracts for sensory-motor crosstalk in healthy and pathological conditions. We include arguments regarding uncertainty in anxiety reactions to the pandemic and derive from our model treatment suggestions which are supported by scientific evidence. Our model is based on systematic control theories and emphasizes the role of goal conflict regulation in health and pathology. We also address anxiety reactions as a spectrum ranging from healthy to pathological coping with uncertainty, and present this spectrum as a transdiagnostic entity in accordance with recent claims and models. 
Conclusions: The human need for controllability and predictability suggests that anxiety disorders reactive to the pandemic's uncertainties reflect pathological disorganization of top-down bottom-up signaling and neural noise resulting from non-pathological human needs for coherence in life.

Keywords: COVID-19, sensorimotor, anxiety disorders, ANS, ascending activating system, descending activation, control theory

\section{INTRODUCTION}

The human mind seeks coherence in life (1). The COVID-19 outbreak exposed the world to a prolonged uncertain situation which in turn poses a risk for increased psychopathology in the general population across ages and increased risk in individuals with pre-existing psychiatric disorders (2-6). A neglected aspect of this pandemic is the mental health suffering of the whole population (people who lost their relatives, out of jobs, familial economy crashes, people confined at home, elder people socially isolated and the worst, uncertainty about the future). Calls for proactive approaches to treat have been published (7).

Anxiety disorders are associated with intolerance of uncertainty $(8,9)$. The highly anxiety-related concept, intolerance of uncertainty, has been historically defined in science in the early 1990s and further developed into a transdiagnostic risk factor suggesting that intolerance to uncertainty fuels reactive anxiety across many disorders (10) and this is evidence-based $(8,11)$. Anxiety disorders have the highest prevalence of all mental disorders, displaying compromised functioning with varied levels of prognosis and remission (12). In addition, treatment resistance or partial remission are prevalent $(13,14)$.

The objective of this review is to identify the brain circuits involved in the human reaction to uncertainties and their connectivity with sensorimotor pathways. Additionally, this review aims to suggest treatment implications for increase of the tolerance to uncertainty based on the identified circuits and their motor counterparts. Our view is of major importance for timely treatment while facing the secondary effects of the COVID-19 outbreak, which is the peaking pandemic of mental health.

The neuroscience of the healthy individual or "the confident brain" shows that in situations of uncertainty distinct neural populations actively compute, in a stepwise manner, the chances for a rewarding experience and the potential costs and create an anticipation which is the guide for adaptive behaviors. This develops from (1) sensory processing, (2) internal state and environmental evaluation, (3) deciding on constancy of input variables, comprising a rule of certainty or uncertainty, and finally (4) anticipating an outcome prediction that results in particular action to be taken. This process involves the human capacity for self-control, which is conceived as the last, active part of the capacity for self-regulation. Self-regulation according to this approach has been defined $(15,16)$ as the ability of the organism to return to baseline after mounting specific responses to an environmental stimulus. Intrapersonal neurobehavioral co-regulation is defined as the capacity of the organism to subordinate all neurobehavioral capacities to enhance learning that allows it to be adaptive to the environmental requirements. It is also defined as the capacity of the organism to return to balance, following adaptation of the enhanced neurobehavioral subsystem to the environmental stimuli $(16,17)$. The operational definition of self-control includes everything that one does in the "operate" phase, which is the "return to balance" phase in the definitions of self-regulation. This is in accordance with classical and well-accepted theories on the human subjective sense of perceived control (18) and the need for predictability to establish and maintaining control (19). The process for coping with uncertainty described above also involves aspects of anticipated cost and reward (20) within the capacity for goal conflict regulation (21).

Goal conflict regulation has been found to be decreased in conditions of anxiety and depression $(22,23)$, suggesting that this capacity may enhance self-control by optimizing predictability and the pathways of cost reward calculations. Thus, self-control, the sense of controllability and calculations of cost and reward through the global function of goal conflict regulation are involved in the healthy process of coping with uncertainty. This has been related to facilitation of the Behavioral Inhibition System's (BIS) (24) reaction by supporting predictability toward making a choice and taking an action. Interestingly, hippocampal and right frontal theta frequencies have been suggested as biomarkers of "healthy anxiety" arising from situations of goal conflict and uncertainty suggesting different types of arousal are responsible for the generation of anxiety in healthy individuals $(21,25,26)$. Higher frequencies such as alpha rhythmicity have been recently reported as appearing at the last stage of the process of goal conflict regulation and suspected as a result of the motor system (27). Anxiolytic action on the behavioral inhibition system implies that multiple types of arousal contribute to anxiety. Accordingly, we argue in this paper for the centrality of motor regions in regulating healthy cognitive processes of uncertainty. It has also been found that the anxiety related to the theta frequency has not been captured by anxiety and depression as assessed by well-accepted inventories (27).

We suggest in this paper a process related to healthy coping and supposedly "healthy anxiety" $(21,24,28)$, which in turn may serve as a lesson for treatment of more pathological cases of anxiety. The centrality of motor regions in encoding and resolving situations of uncertainty is implicated in the existence of a goal or multiple goals requiring an action to be taken as suggested in the global function of goal conflict regulation. The difference between healthy and pathological anxiety in uncertain situations is suggested to be embedded in the existence of 
goals to be regulated and more than that, in the availability of realistic goals to be achieved and that an action toward one of them matters for the resolution of uncertainty. The Sensorimotor-Cognitive-Integration-Circuit (SCIC), described below, is suggested to be the basis for healthy processing of uncertainty.

In the last section of this paper, we suggest the centrality of sensorimotor regions as a target for psychotherapeutic interventions aimed to increase confident cognitions in anxiety disorders during the COVID-19 era.

\section{INTOLERANCE TO UNCERTAINTY IN ANXIETY DISORDERS}

Regular daily life consists of different aspects of certainty and uncertainty. The human mind utilizes anticipation in order to accommodate to new and uncertain situations. In anxiety disorders, this positive anticipation allowing adaptive behaviors is at least partly impaired. A state of intolerance to uncertainty is a core factor in anxiety psychopathology $(29,30)$. We suggest that prolonged situations of uncertainty such as the COVID-19 pandemic may increase the risk of and prevalence for developing anxiety disorders in the general population.

Higher levels of intolerance of uncertainty are associated with internalizing psychopathology, including generalized anxiety disorder, obsessive compulsive disorder, social anxiety disorder, panic disorder, depression, and eating disorders (29, 31). Across disorders, uncertainty is thought to provoke anticipatory anxiety and to result in behaviors that are maladaptive attempts to reduce uncertainty, such as worry, reassurance seeking, checking, and hypervigilance $(32,33)$. In many cases of anxiety disorders, a constant perception of uncertainty without environmental justification is prominent. In others, on which this review focuses, environmental uncertainty elicits, or exacerbates the onset and reoccurrence of anxiety symptoms.

Anxiety disorders are characterized by a wide range of cognitive and somatic symptoms and sufferers have a higher lifetime prevalence of overall dysfunction and co-morbid psychopathology, particularly depression. Lifetime rates of cardiovascular, respiratory, gastrointestinal, and other medical problems are disproportionately high in individuals with anxiety and panic/fear disorders (34). Epidemiological survey findings show that anxiety disorders are the most prevalent mental disorders worldwide and they are associated with significant comorbidity and morbidity $(35,36)$. The WHO recently estimated global mean prevalence for any anxiety disorder, including PTSD, as $21.7 \%$ in the general population (12). Treatment resistance is reported to be also very prevalent $(13,14)$.

We present our view of anxiety-related behavioral, somatic and subjective manifestations from a transdiagnostic perspective. This is supported by the view adapted in the DSM-5 (37) in which the five supposedly distinct axes of the DSM-IV were deleted and the new approach published in 2013 requires the indication of the levels of disorders' severity and need for outside facilitation. In the DSM-IV, anxiety disorders were related mainly to axis 1 although detected in disorders included in axis 2 too. The approach taken by the DSM-5 advises that elicitation and reoccurrence of anxious reactions may appear in a transdiagnostic manner.

We propose that anxiety reactions are a continuum ranging from "healthy anxiety" to pathological anxiety which may be termed as "the anxiety spectrum." The healthy anxiety is defined by reacting to an objective threat with defensive alarmed behavioral and subjective responses which are justified by the existence of the threat $(21,24,28)$. Pathological anxiety depends on the severity of the reaction and the level of negative impact on regular functioning according to the DSM-5.

The transdiagnostic approach has been recently suggested as validated by treatments which were generally outlined and currently prescribed for a wide range of patients with considerable therapeutic broad effects $(28,38,39)$. Additionally, the results of the National Comorbidity Survey (40) which was an epidemiological study, with 65,244 adult participants in the United States aged 15-54 years suggest a small number of "pure" cases and revealed a majority of comorbid cases. Furthermore, this approach determines fewer stigmatic effects and implies a humanistic perspective of a particular individual condition.

We also suggest that pathological anxiety presents a reaction to internal cues, which cause over-arousal and hypervigilance to external cues, not necessarily encoded as a detailed cognition. This view puts forward the pathological matching of internal and external cues in severe cases, suggesting that uncertain situations are transformed into a threat perception by negative anticipations. Furthermore, our transdiagnostic approach is in accordance with the "transdiagnostic model of uncertainty" $(28,39,41,42)$, which suggests that a threat perception is generated when one is confronted with an uncertain situation. In accordance with recent evidence $(8,10,11)$ we also suggest that the matrix of the intolerance to uncertainty axis by the anxiety reactions axis builds up to extremity and severity via bi-directional effects of intolerance to uncertainty on anxious reactivity and vice versa.

From a cognitive perspective, people with anxiety disorder translate the ambiguous situation into a threat, thus the topdown bottom-up cerebral connectivity produces a fear reaction (43). From a neurochemical angle a stress reaction is recruited resulting from the fear cognition and the threat perception (4446). From a peripheral perspective, sympathetic over-arousal is apparent including peripheral reactions such as psychogenic tremor, perspiration, increased heart rate, respiration and gastrointestinal symptoms (47).

Neural noise the random intrinsic electrical fluctuations within neuronal networks which are not associated with encoding a response to internal or external stimuli; [e.g., (48, 49)]\} has been suggested to be involved in the reactions of individuals with anxiety disorder to uncertainty $(30,50,51)$. Neural noise may be the basis of an exaggerated anxiety reaction. Neural noise may result from over-reaction to alarming peripheral cues without a clear cognitive estimation or evaluation of a threat. Therefore, competing negative signals to the cortex may not be processed accurately in a condition in which an internal peripheral alarming cue meets external potentially threatening stimuli and this assembly combat in neural circuits 
occurs without a time limited resolution within the cortical descending order. This may be part of the sympathetic reactivity and the basis for the peripheral symptoms (52).

In this respect, neural noise represents the competition between multiple intrinsic and extrinsic driven signals, which carry various types of negative information. This may block the cortical proficiency to attribute a clear cause to the signals thus no goal-oriented resulting decision on an action to be taken is available as the facilitating encoding is lacking. It is predominantly a neurological state encompassing a reduction in cortical production of encoded cognitions. In uncertain situations, the salience of a general negative experience generated by internal somatosensory systems produces neural noise which may also block the availability of other sensory inputs as they become available, including signals which carry relaxing information, thus increasing and prolonging, even fixating, the risks for anxious reactions. Thus, the subjective sense of anxiety in uncertain situations may be experienced as objectively driven even though internal cues are heavily involved and objective threats may be overly evaluated as costly in this condition.

We suggest a model of complex brain cyclicity, emphasizing the role of sensorimotor regions in the generation of certainty vs. uncertainty cognition. Of special interest of this review is the issue of autonomic nervous system (ANS) coupling with the cortex to address peripheral reactions of anxiety disorders to uncertainty beyond a simple distorted cognition as widely accepted.

\section{A BIOLOGICAL MODEL FOR THE "CONFIDENT BRAIN"}

The healthy cognitive coping with uncertainty or the "confident brain" employs a stepwise process using (1) sensory processing; (2) state and environmental evaluation; (3) recognizing whether a rule of certainty exists or rather a rule of uncertainty and finally (4) outcome prediction (50), see Figure 1. Recent data show that this process of uncertainty evaluation and resulting reaction involves top-down and bottom-up cyclical brain modulation in numerous regions, including sensorimotor regions, the limbic system, the reward circuits and frontal regions responsible for executive functions $(53,54)$.

From a neurochemical perspective, noradrenaline influences learning of uncertain events arising from unexpected changes in the environment (55). In contrast, acetylcholine balances attribution of uncertainty to chance fluctuations within an environmental context, defined by a stable set of probabilistic associations, or to gross environmental violations following a contextual switch (55). Dopamine supports the use of uncertainty representations to engender fast, adaptive responses (55). Diurnal cortisol is disrupted in prolonged uncertain situations (56) and in anxiety disorders (57). Various studies support the role of dopamine and serotonin in anxiety disorders (58-60).

From a peripheral perspective, healthy participants reacted to uncertainty with altered spinal reflexes $(30,61)$ and gastrointestinal symptoms (62-64). Uncertainty related sympathetic over-arousal has also been indicated $(52,65)$.
The thalamo-cortico-striatal circuit (TCS) is thought to be central in the pathophysiology of anxiety disorders (66) in addition to the amygdala and the corticolimbic system (67, 68). We suggest a more complex circuit for the impact and processing of uncertainty, which is hampered in anxiety-related disorders. Dysregulated coupling of the ANS with the cortex in anxiety disorders (69) may be shown in the compromised function of the tracts of the crus cerebri traveling from the cortex to the spinal cord, filtering information en passage (70). The coupling of the autonomous system is also supported by ascending tracts reaching sensorimotor cortices through filtering information by the basal ganglia and amygdalae (7173). Specifically, microstructural differences in the bilateral corticospinal tracts (CSTs) were detected among patients with anxiety and depression $(66,74)$. Lower fractional anisotropy (FA) was found in the white matter of the CST. Further, increases in FA in the CST-related fiber paths of the bilateral posterior limbs of the internal capsule, right superior corona radiata, and the left external capsule were found while an increase in CST excitability has been detected in participants exposed to fearful images (75). The CST has been shown to have a central role in a more complex circuit for evaluation of uncertainty and tolerance to ambiguity in anxiety disorders. Changes in gray matter in the basal ganglia and in white matter in the corticospinal tract were reported in anxiety disorders (66). In over-arousal and over-stimulated conditions the corticospinal neurons' function is inhibitory through their affinity to GABAergic and glycinergic presynaptic function (76). Both basal ganglia and the corticospinal tract which were historically accepted as motor regions, have been recently recognized as involved in cortical and subcortical filtering of information that is transmitted to and commanded by the prefrontal and somatosensory regions (77). Thus, the link between sensory integration and motor output, the input and output of healthy processing of uncertainty (50), operate in a topdown bottom-up manner. This involves commands to peripheral regions such as those ending in spinal reflexes as well as posing significant impact on cognitive and emotional processes (72). We suggest that ascending sensory and descending corticospinal tracts are integrated in the somatosensory cortex and that they selectively project to the prefrontal cortex following filtering by the basal ganglia and the amygdalae. It is further suggested that in anxiety disorders these neural connections are hampered (78-82), see Figure 2.

Our model complies with the rules of Control Theory, developed from Maxwell's mathematics (83) and utilized in engineering $(84,85)$. The first applications to Psychology are found as early as the 1940's in the works of Craik (86), Wiener (87), and Ashby (88). Since the 1990's, Powers' (89) application of Control Theory to human goal-oriented behavior and his development of perceptual control conceptualization termed as Perceptual Control Theory (PCT) has gained renewed scientific attention (90) while previously regenerated in the form of a selfregulation theory, [e.g., $(18,91,92)$ ] relating to Cannon's view on homeostasis (93) and Wiener's (87) theory on Cybernetics as essential parts of it.

Control Theory implies discrepancy-reducing feedback loops for correction of error signaling by various controllers' sensors in 


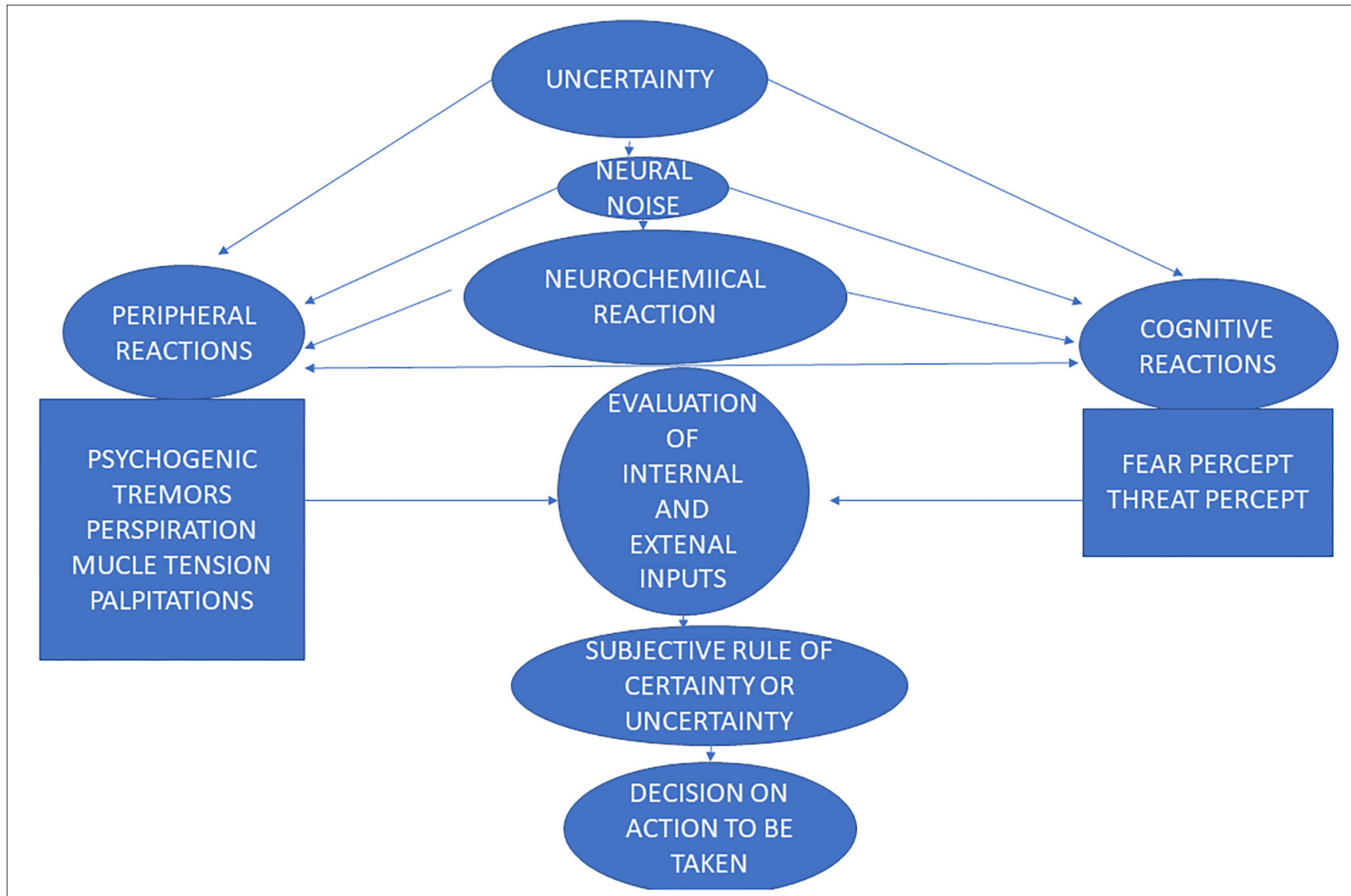

FIGURE 1 | The process of uncertainty from the stage of sensory input to the stage of making a decision on an action to be taken, in anxiety disorders.

each of the sub-systems which together activate the entire control system. PCT implies a hierarchy of eleven modules, ranging from sensory input "intensity" (28) to personal transcription of "programs," "principles," and "systems," which are the highest modules (94) ending in the organization of the individual's principles in systematic personal organization, while all modules are functioning in a top-down bottom-up manner to elucidate a perception for a goal directed behavior elicitation. These modules maintain feedback loops and error correction signals (termed as internal reference standards) within each loop and each module as well as between modules for an overall perception on a goal directed behavior. Recently this theory has been applied to healthy coping with uncertainty suggesting the "The Effort Intensity Continuum" developing from avoidance to toleration, coping, and embracing. McNaughton and Gray (21), Gray (24), Gray et al. (25), Einstein and Mansell (28), and Gray (95) describe how a central comparator system may trigger a pause in an operating program within multiple trials to resolve the mismatch between the perceived threat (cost) and the individual's desired outcome during defensive approach (expected reward). The term reorganization has been suggested by Powers (89), to be apparent as a trial and-error learning process leading to the resolution of this mismatch conflict in uncertain situations.
Carey (96) suggests that this phase of reorganization requires sustained attention by the systems responsible for encoding the goal conflict toward reaching a resolution, which he agrees is a decision on an action to be taken as we argue in this paper.

Our model also is in accordance with the Perceptual Control Theory (PCT) (41) which proposes, in line with the mathematical Control Theory formulations, that the nervous system as a whole is an organized control system in which higher systems receive feedback from lower systems, describing a cascade of levels. Accordingly, we suggest that the neurological pathways identified as central for coping with uncertainty along the central nervous system (CNS), are fed back in a top-down bottom-up manner, within a cyclical control system.

A good example for that is our claim that although sensory inputs start the process of coping, our model suggests that the sensory inputs by the ascending tracts may be transformed or even corrupted (97) in cases of prior cognition, especially if there is a sensory-input-opposing-cognition. Thus, the starting signal may be sensory (bottom-up) in healthy cases and cognitivedistorted (top-down) in more pathological cases of coping with uncertainty including the exaggeration of negative (cost) view of it. Therefore, while processed on the continuum of the feedback loops, a particular sensory input may affect the descending tracks 
with a deteriorating error implying a destructive, not correctly computed signal. In such a case, the decision on the action to be taken may not be useful for reducing uncertainty. This could potentially explain pathological coping with uncertainty and its aligned increased anxiety.

It is suggested in our model that signals occur "on the fly" throughout the process and contribute to its continuation with a healthy, realistic, and achievable goal of an action to be taken. This is in accordance with PCT's main feature, explicitly modeling the control of sensory input. This is contrary to previous computational models of the mind which imply precise predictions as well as a mindful mapping to support control of the individual's behavior and the outside world prior to an action (41). That is, healthy coping with uncertainty respects sensory inputs as they become available and does not intend to override them with a-priori cognitions. This is modulated by a healthy ANS. However, the role of higher cortical regions such as the sensorimotor areas, in healthy cognition creation for a decision on an action to be taken, is argued here in accordance to the PCT, to be within the circuitry continuum of the multivariate error correcting and feedback processing.

\section{THE SENSORIMOTOR AREAS' CONNECTIVITY WITH THE AMYGDALA}

Likelihood uncertainty is represented in the associated sensory pathway (visual). In contrast, prior uncertainty is represented in putamen, amygdala, insula, and OFC (98).

Resting state (rs)-fMRI studies show alterations in the amygdala-sensory/(pre)motor pathways and suggest that these alterations may be involved in psychiatric conditions (99). Specifically, others suggest recently that the amygdala and bed nucleus of stria terminalis (BNST) connectivity are neural markers of anxiety disorders. Whereas, amygdala-thalamus/ACC rs-functional connectivity supports adaptive regulation of threat response in healthy controls, BNST-caudate rs-functional connectivity may reflect maladaptive neural processes that are dominated by anticipatory anxiety (100).

\section{THE SENSORIMOTOR AREAS AND THE FILTERING OF THREAT COGNITIONS BY THE BASAL GANGLIA AND AMYGDALA}

Classically, the basal ganglia have been considered to have a role in producing habitual and goal-directed behaviors. Recently evidence indicates that the basal ganglia are also involved in neural and behavioral inhibition in both goal-directed and habitual choice-preference motor actions mediated by fronto-striato-subthalamic-pallido-thalamo-cortical networks. Imbalance between goal-directed and habitual action and inhibition has been suggested as involved in manifestations of neuropsychiatric disorders (101). Thus, the inhibitory role of the basal ganglia over motor outputs resulting from reactions to uncertainty, suggests that it filters signals to motor cortices involved in reactions to uncertainty such as the sensorimotor regions. These inhibitory signals may be available to the cortices through excitatory terminals in the striatum (101) raising the hypothesis that competing signals of inhibition (threat cognition) and excitation (reward cognition) are imbalanced in conditions of intolerance to uncertainty, such as evident in anxiety disorders which in turn may end in erroneous expectations and less than evidence-based motor outputs of a decision.

\section{THE SENSORIMOTOR AREAS AND COGNITIONS OF REWARD AND ASSURANCE}

Recent studies suggest an important role of the basal ganglia in reward expectation (102), a missing component in anxious intolerance of uncertainty. Sensorimotor/cognitive activities of neurons in the basal ganglia are strongly modulated by expected reward. While signaling to the brainstem, the basal ganglia may affect the CST too, which in turn connects the brainstem to the cortices and couples the ANS to them. Thus, peripheral reactions in anxiety conditions resulting from intolerance to uncertainty may be based on dysfunction of the differential signaling of the basal ganglia. Neurons in the caudate nucleus and the substantia nigra pars reticulata are extremely sensitive to differences in expected reward. Therefore, they lead to a bias in excitation/inhibition between the superior colliculi suggesting that motor integration toward a reward could occur more quickly or slower (103). Therefore, these results may explain the approach-avoidance (24) conflict in anxiety conditions when faced with a probability of reward ending in a threat perception and avoidance behavior because of negative interpretations of rewards that are not certain. It is suggested that the reward modulation occurs in the caudate where cortical inputs carrying spatial signals and dopaminergic inputs carrying reward-related signals are integrated. This suggests the centrality of the basal ganglia sensorimotor cognitive neurons in filtering of the competing signals to the motor cortices and through its connectivity all the way to the CST via its signals to the brainstem.

\section{THE "EMOTIONAL" AND "AFFECTIVELY CONSCIOUS" BRAINSTEM AND ITS ROLE IN SOMATOSENSORY INTEGRATION AND MOTOR OUTPUT}

Affective Neuroscience's approach suggests that affective consciousness is generated and processed by sub-cortical areas (104). Accordingly, the "emotional brainstem" includes three major networks-Ascending, Descending and Modulatory (105-107). The brainstem is a somatosensory-motor integrating gateway for emotional and consciousness regulation. The ascending network includes the spinothalamic tracts and their projections to brainstem nuclei. The Descending motor network includes medial projections from the reticular formation and modulation of signaling impacting emotional salience, and other lateral projections to higher regions such as periaqueductal gray, hypothalamus, and amygdala that integrating behavioral actions resulting from emotional regulation. The brainstem 


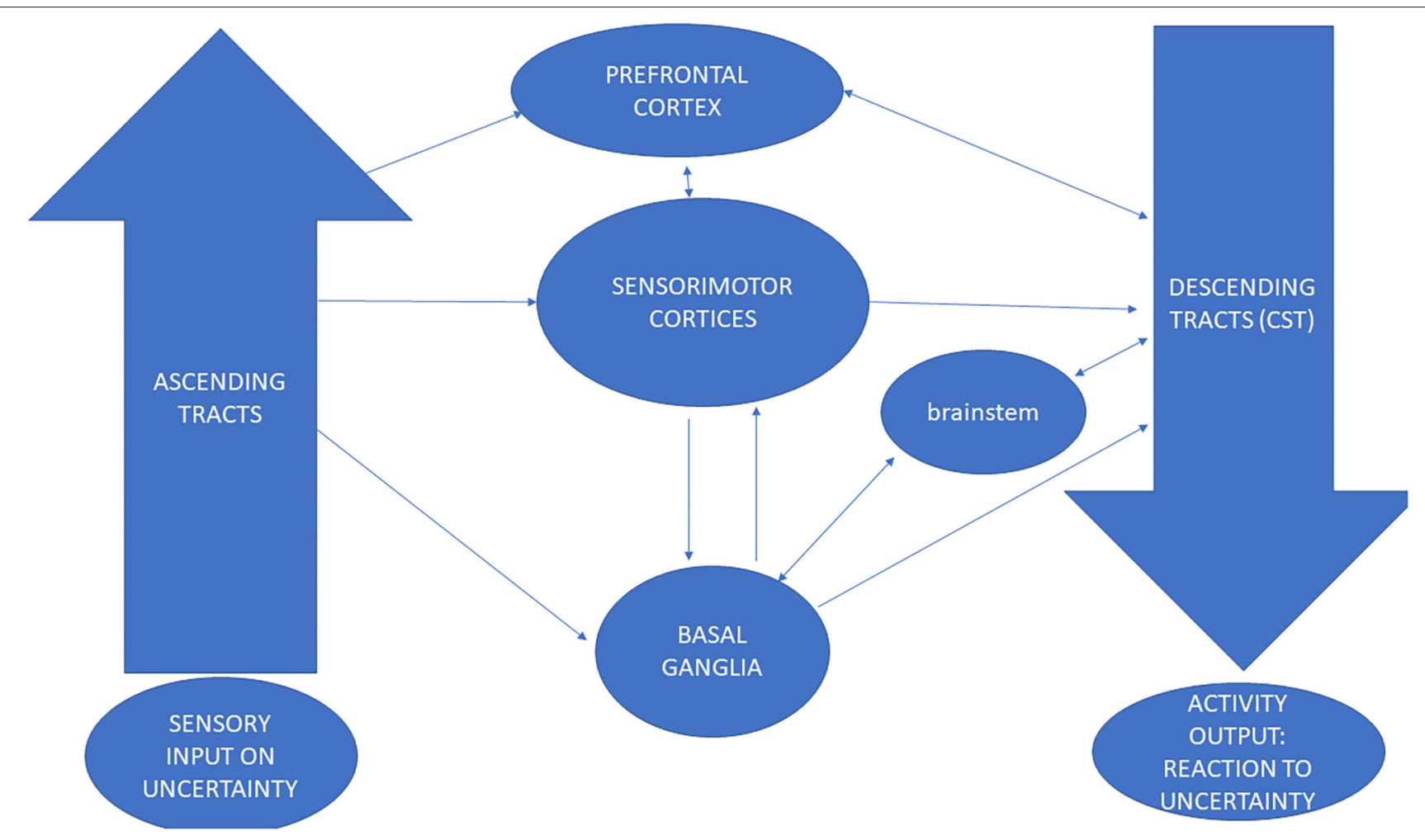

FIGURE 2 | The involvement of sensorimotor circuits in processing and filtering uncertainty cognitions in the healthy brain: The Sensorimotor-Cognitive-Integration-Circuit (SCIC).

regulatory functions are prominent through neurotransmitter pathways including the serotonergic raphe nuclei expression of serotonergic synapses, the connectivity to ventral tegmental area dopaminergic expression and locus coeruleus synaptic effects of noradrenergic activity. All these neurotransmitters are coordinated through different loci in the brainstem. Phylogenetically older brainstem networks work in a caudal to rostral manner with bidirectional signaling to evaluate sensory information and modulate somatosensory inputs including those which are peripherally generated. Thereafter, the brainstem triggers neurobehavioral action patterns (105-107). Ontogenetically, the brainstem and spinal cord develop within two separated plates parted by the sulcus limitans in the brain's fourth ventricle. This ventricle separates the alar plate, from which sensory neurons emerge and the basal plate, from which the motor neurons arise (108). The CST as the largest connection of the cortex to the brainstem is suggested as the main pathway for integration of sensory input toward an action to be taken.

\section{PROGNOSTIC CONSIDERATION OF PSYCHIATRIC CONDITIONS RESULTING FROM THE COVID-19 SITUATION}

Sensorimotor activity in anxiety disorders may be habitual or peripherally dysregulated while goal-directed actions may include mainly activities aimed at reducing the sense of anxiety and increasing the sense of certainty. As previously suggested (21, 41 ), we argue that goal regulation by error correction aligned to accumulating sensory input characterizes the healthy coping with uncertainty, while goal conflict is typically evident in pathological cases of severe anxious reaction in uncertain situations, in accordance with earlier findings $(25,26)$. Additionally, we propose that the maladaptive coping with- and intolerance to uncertainty is a condition in which the lack of specific achievable goals is apparent. In this condition, small achievable goals do not reach a high expected rewarding value and the individual is aiming at a global, beyond his/her control, goal. For example, a goal of absolute protection from the pandemic or the wish that the pandemic would be shorter or readily over.

We argue that the lack of specific achievable goals, may result from the predominantly neurological condition of neural noise which prevents a clear cognitive attribution to a cause, thus inhibiting cortical descending order toward a motor output, namely, an action to be taken. This condition precludes a resolution, extending, and exaggerating the anxious reaction to uncertainty. That is, goals defined unclearly imply the diffusion of actions. This diffusion of action in turn, results from the build-up of the intolerance to uncertainty and anxiety matrix $(8,10,11)$ into severity and extremity in a bi-directional manner. These anxiety-related diffused actions may become a challenge during the COVID-19 era, which consists of real threats and 
uncertainties. We aim to suggest a treatment approach which may target the bi-directional pathways of perceptual tendencies in anxiety disorders and sensorimotor modulation to increase the tolerance to uncertainty by the selection of achievable goals prior to working toward a resolution. Selection of goals is considered by PCT an input and as such it is of therapeutic merit $(109,110)$.

The sense of control is closely associated with reduction in anxious reaction to a given situation $(111,112)$. The need for general control is based on the human need for coherence in life (1). Thus, we argue that the pathological intolerance to uncertainty exhibited in anxiety-related disorders originates from the non-pathological need for controllability and predictability. This need is highly challenged during the pandemic. We aim to suggest a pathway for stimulating motor actions modulated by positive cognitive inputs, which of course could not control the COVID-19 entire situation but may enhance a partial sense of control and sense of coherence in the anxious individuals' personal life during the pandemic. We will outline manners for reactivation of sensorimotor systems within the limits of predictable outcomes to release the individual from the pathological automatic and habitual motor actions, which lack the resulting sense of control. This will allow filtering of sensory inputs and motor outputs, which may result in a bi-directional mode of cognitive driven motor actions affording partial control and more accurate and positive prediction. As emotional survival is threatened by the entire COVID-19 situation, we suggest that the threatened individual has "always something to do about something" meaning partial control in predictable situations and familiar human circles. This line of defense is supported by results of heightened perceived loneliness during the pandemic which attests to a subjective common experience not necessarily depending on physical loneliness (113).

For prognostic considerations, uncertainty requires analysis and treatment of insecurities in the patient's social support circle and the therapeutic definition of the particular unrewarding experiences and percepts and individual translation of uncertainty. Both acute and chronic anxiety conditions should be targeted from a peripheral perspective too and not only from a top-down brain regional view as human cognition of uncertainty in anxiety disorders is associated with bodily sensations. Regulation of physical sensations may support the rise of positive anticipations in uncertain situations and conditions. In anxiety disorders this could be supported and achieved by short-term techniques targeting control over bodily sensations along with changes in anticipatory cognitions. The combination between social support and sensorimotor control is required as simple correction of a negative expectation in uncertain situations may relapse with potential reoccurrence of the negative percept and with sympathetic over arousal in cases in which interpersonal resolution and reassuring social support are lacking.

From a prevention perspective, uncertainty acceptance as an inevitable part of everyday life may increase resilience, support the availability of positive anticipations, and turn the anxiety disorder into the "confident brain." This requires wider boundaries for creation of a negative perception and a higher threshold for negative expectation as suggested in the Drift Diffusion Model (114). This can allow the anxious individual to promote tolerance to uncertainty and reduce pathological motor activity resulting from threat perception of uncertainty. These wider boundaries (114) are associated with more confidence and less reward prediction error (115). We suggest that slowing down of automatic and habitual motor activity may support better excitation-inhibition balance within the junctions of sensorimotor circuits that recently have been shown to affect cortices and cognition as well as connectivity to the ANS.

By supporting anxious individuals during the COVID-19 situation to adopt wider boundaries for a decisive motor action as the first line of defense of treatment, those individuals may be able to perceive that the threat (infection and death of oneself and relatives) is not immediate and the reward (the pandemic will end as all previous pandemics did in history and the entire globe will get back to normal life) is out there in the future. These more adaptive perceptions may free the brain from pathological frequent motor reactions, which are part of the burden of anxiety disorders.

From a Control Theory view $(84,85)$, error signals emerge from the comparison of the actual value to the expected value and are organized as such. Faulty signals are disorganized, representing a faulty sensor of a controller, that is, neural noise and a pathological condition. In accordance with Powers (116) who suggested the term "reorganization" for describing the error correcting feedback loops process, we propose that an organized control system with systematic continuous error correction (healthy state) may turn into a disorganized control system which consists of sensors generating faulty signals (neural noise, pathological state) when faced with uncertain situations.

Only the comparison between faulty and reliable sensors could bring about an organized perception for a goal-oriented action to be taken. Setting a goal with the patient may work as an input to the cortex $(109,110)$, enabling cortical capacities for an overriding command and recruitment of sensorimotor regions. To support this view, it has been suggested in modeling the PCT, that higher regions act more rapidly than lower levels $(109,110)$. Thus, the cortex's ability includes the capacity for a prevailing command. Its representation is reflected in the transformed responses of the subcortical regions.

We note that assignment of goals, even in fantasy or imagination, is an input to the cortex and a goal is generated by the extent of the error signal (117). Lack of intention characterizes pathological conditions and a state of subcortical regions overtake resulting in incidental actions which are not clearly oriented. That is, selection and assignment of a goal and an intention in conditions of a disorganized system represents cortical transformation of the input-output balance to an organized state. We suggest that this way the cortex is able to activate a predominant descending command to reorganize the entire system including peripheral reactions as well as the upstream collection of ascending signals which were blocked in the disorganized state. According to Control Theory, the response signals from subcortical regions of the human control system to the cortical command represents the transformative dynamics of the command into appropriate $\backslash$ required signals 
of comparison. The subcortical regions are pre-programmed to respond to such a prevailing command. Pre-programing of subcortical regions develops early in fetal and infancy life through rostral to caudal and basal to alar trajectories while the developing control of the cortex over the brainstem is dependent on CST maturation $(118,119)$.

The faulty signals from a disorganized sensor distort external cues and overwhelm cortical input with internal disorganized signals thus preventing encoding and increasing subjective perception of uncertainty. In this sense revival of attachment links including past and present emotional securities may achieve the aim of computing a cortical command in conditions of disorganization.

The term "transforming command" in Control Theory represents in our view the human organism's capacity for fantasy and imagery. Accordingly, an assignment of a goal can be initiated in fantasy and imagery first. This is evident in the technique of guided imagery, in creative functions and in the therapeutic effects of attachment links' past intensities and sensations retrieval.

Beyond saving the CNS from faulty sensors, the human fantasy also encompasses the human capacity to go further and to transform the human control system into an accomplishing system rescuing the human organism from redundancy. Therefore, the preponderant cortical command is available to transform the human control system for negative and positive reasons, while the aligned actions to be taken, or results, in Powers's terms, are healthier when an error signal shows that the expected value is higher than the actual one. This condition may take the "confident brain" to further achievements while maintaining emotional survival in a stepwise multiple transformation of the human control system by a successive selection of goals. However, this positive and healthy process is also dependent on the error signal and internal reference standards for recruitment of cortical command and the dynamics of the subcortical transformed signal responses.

As Powers $(89,116)$ noted, error signals generated by higher regions comparing internal reference standards to the actual values of the perception allow the retrieval of past stored signals from lower regions. Thus, revival of attachment links may contribute to the restoration of organized error correcting and feedback top-down bottom-up signaling for a goal-directed action integrated by sensorimotor regions in a more organized manner, and may also turn pathology into a healthy process of growth further than the original aim of emotional survival in uncertain situations. This implies a shift in past personal programing, principles and systems, which organize the individual's principles, the highest modules in the PCT (94), and eliminates linear causality and forced negative predictions from any pathological condition. This proposition is in accordance with our view of the "anxiety spectrum," a continuum ranging from "healthy anxiety" to pathology.

Providing increased security in close relationships during the COVID-19 situation may enhance excitation-inhibition balance within sensorimotor-driven cognitions of uncertainty. According to Bowlby (120) and Ainsworth (121), the sense of attachment security in close relationships (confidence that one can trust on relationship partners and that they will be available and supportive when needed) encourages relaxed exploration of new, unusual information and phenomena, and favors the formation of open and flexible cognitive structures. Being confident in their ability to deal with uncertainty, people who hold a sense of attachment security might be able to incorporate new information (sensory input) at the expense of temporary perplexity or confusion, reflecting "the confident brain." Cognitive uncertainty might not generally threaten their sense of competence, lovability, and control. Rather, they might realize that perplexity, like other challenging experiences, is short-lived and can lead to greater mastery and broaden their sense of coherence and meaning (122) thus suggesting adaptive sensorimotor control.

In support of this view, there is extensive evidence showing that the sense of attachment security in close relations is closely related to heightened tolerance of uncertainty, lower levels of dogmatic thinking, and less rejection of information that challenges the validity of one's beliefs (123-125). Moreover, dozens of studies summarized by Mikulincer and Shaver (122) have consistently found that the sense of attachment security is associated with lower levels of anxiety and distress during and after exposure to stressors and less prevalence of anxiety disorders. Therefore, another line of defense to reduce anxiety in the COVID-19 situation might involve making contextually salient the close relations that a person feels securely attached to, or priming mental representations of attachment security in these relationships, using guided imagination exercises or structured autobiographical memories retrieval (126). This could potentially allow decisions on motor actions within more narrow boundaries and less prediction errors. The positive cognitions related to attachment security ("I'm worthy of love," "Others are benevolent and trustworthy") may afford decisive motor actions to protect the close others that provide emotional security $(127,128)$.

As a neurobiological background for the expected efficacy of these suggested treatments, which consider sensory input regarding the long-term sense of security to modulate habitual and automatic anxious reactions during times of change and uncertainty, we note that others found in fMRI sequential inference tasks multivariate patterns of activity representations that changed more rapidly during periods of uncertainty following a change in behavioral context. In motor cortex, this phenomenon was indicative of discontinuous change in behavioral outputs, whereas in visual regions, the same basic phenomenon was evoked by tracking of salient environmental changes. These results may provide a dynamic substrate for healthy learning that facilitates rapid disengagement from learned motor actions during periods of change (129). Furthermore, flexibility of boundaries in a context-specific manner, suggested here as a treatment technique, has been shown to buffer psychopathology during the COVID-19 situation (130). Taken together, retrieval of long-term solid sense of security and a build-up of boundaries' flexibility regarding a decision on an action to be taken, may increase the tolerance to uncertainty and provide a sense of certainty in controllable daily affairs with one's significant others, which in turn is thought to improve the status of individuals with anxiety disorders during the pandemic using the unique modulation of cognitions by sensorimotor regions. 


\section{CONCLUSIONS}

We suggest to extend the understanding of intolerance of uncertainty in anxiety-related disorders by the SensorimotorCognitive-Integration-Circuit (SCIC). This assertion goes hand in hand with recent views suggesting that sensorimotor regions modulate cognitions. During the COVID-19 pandemic the issue of uncertainty gained much scientific attention. We add to this body of knowledge specific treatment suggestions based on Attachment Theory to shield the effects of the pandemic's uncertainties on emotional survival and on the tendency to collapse to anxious automatic and habitual motor reactions generated by negative cognitions due to lack of general control. According to our view, emotional security is generating emotional survival, a situation in which a human control system is organized. This in turn supports goal-oriented actions modulated via sensorimotor regions for the availability of inputs and outputs to the cortical perceptual functions and desired results of actions.

\section{REFERENCES}

1. Cervone D, Shoda Y (editors.). The Coherence of Personality: Social-Cognitive Bases of Consistency, Variability, and Organization?. New York, NY: Guilford Press (1999).

2. Rettie H, Daniels J. Coping and tolerance of uncertainty: predictors and mediators of Mmental health during the COVID-19 pandemic. Am Psychol. (2020) 76:427-37. doi: 10.1037/amp0000710

3. Solé B, Verdolini N, Amoretti S, Montejo L, Rosa A, Hogg B, et al. Effects of the COVID-19 pandemic and lockdown in Spain: comparison between community controls and patients with a psychiatric disorder. preliminary results from the BRIS-MHC STUDY. J Affect Disord. (2021) 281:13-23. doi: 10.1016/j.jad.2020.11.099

4. Elsharkawy NB, Abdelaziz EM. Levels of fear and uncertainty regarding the spread of coronavirus disease (COVID-19) among university students. Perspect Psychiatr Care. (2020) 1-9. doi: 10.1111/ppc.12698

5. Voitsidis P, Nikopoulou VA, Holeva V, Parlapani E, Sereslis K, Tsipropoulou $\mathrm{V}$, et al. The mediating role of fear of COVID-19 in the relationship between intolerance of uncertainty and depression. Psychol Psychother Theory Res Pract. (2020) 1-10. doi: 10.1111/papt.12315

6. Parlapani E, Holeva V, Nikopoulou VA, Sereslis K, Athanasiadou $\mathrm{M}$, Godosidis $\mathrm{A}$, et al. Intolerance of uncertainty and loneliness in older adults during the COVID-19 pandemic. Front Psychiatry. (2020) 11:842. doi: 10.3389/fpsyt.2020.00842

7. Keng A, Brown EE, Rostas A, Rajji TK, Pollock BG, Mulsant BH, et al. Effectively caring for individuals with behavioral and psychological symptoms of dementia during the COVID-19 pandemic. Front Psychiatry. (2020) 11:573367. doi: 10.3389/fpsyt.2020.573367

8. Gu Y, Gu S, Lei Y, Li H. From uncertainty to anxiety: how uncertainty fuels anxiety in a process mediated by intolerance of uncertainty. Neural Plast. (2020) 2020:1-8. doi: 10.1155/2020/8866386

9. Wever M, Smeets P, Sternheim L. Neural correlates of intolerance of uncertainty in clinical disorders. J Neuropsychiatry Clin Neurosci. (2015) 27:345-53. doi: 10.1176/appi.neuropsych.14120387

10. McEvoy PM, Hyett MP, Shihata S, Price JE, Strachan L. The impact of methodological and measurement factors on transdiagnostic associations with intolerance of uncertainty: a meta-analysis. Clin Psychol Rev. (2019) 73:101778. doi: 10.1016/j.cpr.2019.101778

11. Gillett CB, Bilek EL, Hanna GL, Fitzgerald KD. Intolerance of uncertainty in youth with obsessive-compulsive disorder and generalized anxiety disorder: a transdiagnostic construct with implications for phenomenology and treatment. Clin Psychol Rev. (2018) 60:100-8. doi: 10.1016/j.cpr.2018.01.007

\section{DATA AVAILABILITY STATEMENT}

The original contributions generated for the study are included in the article/supplementary material, further inquiries can be directed to the corresponding author/s.

\section{AUTHOR CONTRIBUTIONS}

SGF wrote the initial draft of the paper and the revised versions. All authors contributed to editing various versions of the paper as it evolved and approved the final version.

\section{ACKNOWLEDGMENTS}

The authors thank Prof. Haim Belmaker for his careful review and contribution to this manuscript.

12. Charlson F, van Ommeren M, Flaxman A, Cornett J, Whiteford H, Saxena S. New WHO prevalence estimates of mental disorders in conflict settings: a systematic review and meta-analysis. Lancet. (2019) 394:2408. doi: 10.1016/S0140-6736(19)30934-1

13. Bystritsky A. Treatment-resistant anxiety disorders. Mol Psychiatry. (2006) 11:805-14. doi: 10.1038/sj.mp.4001852

14. Roy-Byrne P. Treatment-refractory anxiety; definition, risk factors, and treatment challenges. Dialogues Clin Neurosci. (2015) 17:191-206. doi: 10.31887/DCNS.2015.17.2/proybyrne

15. Ferber SG. Biofeedback mechanisms between shapeable endogen structures and contingent social complexes: the nature of determination for developmental paths. Behav Brain Sci. (2009) 32:392-3. doi: 10.1017/S0140525X09990197

16. Ferber SG. The concept of coregulation between neurobehavioral subsystems: the logic interplay between excitatory and inhibitory ends. Behav Brain Sci. (2008) 31:337-8. doi: 10.1017/S0140525X08 004123

17. Ferber SG, Makhoul IR. The effect of skin-to-skin contact (Kangaroo Care) ahortly after birth on the neurobehavioral responses of the term newborn: a randomized, controlled trial. Pediatrics. (2004) 113:85865. doi: $10.1542 /$ peds.113.4.858

18. Carver CS, Scheier MF. Control theory: a useful conceptual framework for personality-social, clinical, and health psychology. Psychol Bull. (1982) 92:111-35. doi: 10.1037/0033-2909.92.1.111

19. Lazarus R, Folkman S. Stress, Appraisal, and Coping. New York, NY: Springer (1984).

20. Ferber SG, Weller A, Yadid G, Friedman A. Discovering the lost reward: critical locations for endocannabinoid modulation of the cortico-striatal loop that are implicated in major depression. Int J Mol Sci. (2021) 22:1867. doi: 10.3390/ijms22041867

21. McNaughton N, Gray JA. Anxiolytic action on the behavioural inhibition system implies multiple types of arousal contribute to anxiety. J Affect Disord. (2000) 61:161-76. doi: 10.1016/S0165-0327(00)00344-X

22. Moberly NJ, Dickson JM. Goal conflict, ambivalence and psychological distress: concurrent and longitudinal relationships. Pers Individ Dif. (2018) 129:38-42. doi: 10.1016/j.paid.2018.03.008

23. Kelly RE, Mansell W, Wood AM. Goal conflict and well-being: a review and hierarchical model of goal conflict, ambivalence, selfdiscrepancy and self-concordance. Pers Individ Dif. (2015) 85:21229. doi: $10.1016 /$ j.paid.2015.05.011

24. Gray JA. Perspectives on anxiety and impulsivity: a commentary. J Res Pers. (1987) 21:493-509. doi: 10.1016/0092-6566(87)90036-5 
25. Gray JS, Ozer DJ, Rosenthal R. Goal conflict and psychological well-being: a meta-analysis. J Res Pers. (2017) 66:27-37. doi: 10.1016/j.jrp.2016.12.003

26. Nash K, McGregor I, Prentice M. Threat and defense as goal regulation: from implicit goal conflict to anxious uncertainty, reactive approach motivation, and ideological extremism. J Pers Soc Psychol. (2011) 101:1291301. doi: 10.1037/a0025944

27. Neo PSH, Tinker J, McNaughton N. Goal-conflict EEG theta and biased economic decisions: a role for a second negative motivation system. Front Neurosci. (2020) 14:342. doi: 10.3389/fnins.2020.00342

28. Einstein DA, Mansell W. The relevance of uncertainty and goal conflict to mental disorders, their prevention and management: a unifying approach. Cogn Behav Ther. (2016) 9:1-9. doi: 10.1017/S1754470X16000234

29. McEvoy PM, Mahoney AEJ. To be sure, to be sure: intolerance of uncertainty mediates symptoms of various anxiety disorders and depression. Behav Ther. (2012) 43:533-45. doi: 10.1016/j.beth.2011.02.007

30. Grupe DW, Nitschke JB. Uncertainty and anticipation in anxiety: an integrated neurobiological and psychological perspective. Nat Rev Neurosci. (2013) 14:488-501. doi: 10.1038/nrn3524

31. Brown M, Robinson L, Campione GC, Wuensch K, Hildebrandt T, Micali $\mathrm{N}$. Intolerance of uncertainty in eating disorders: a systematic review and meta-analysis. Eur Eat Disord Rev. (2017) 25:329-43. doi: 10.1002/erv.2523

32. Tanovic E, Gee DG, Joormann J. Intolerance of uncertainty: neural and psychophysiological correlates of the perception of uncertainty as threatening. Clin Psychol Rev. (2018) 60:8799. doi: 10.1016/j.cpr.2018.01.001

33. Dugas M, Buhr K, Ladouceur R. The role of intolerance of uncertainty in etiology maintenance. - PsycNET. In: Heimberg R, Turk C, Mennin D, editors. Generalized Anxiety Disorder: Advances in Research Practice. New York, NY: Guilford Press (2004). p. 143-63.

34. Meuret AE, Tunnell N, Roque A. Anxiety disorders and medical comorbidity: treatment implications. Adv Exp Med Biol. (2020) 1191:23761. doi: 10.1007/978-981-32-9705-0_15

35. Locke A, Kirst N, Shultz CG. Diagnosis and management of Generalized Anxiety Disorder and Panic Disorder in adults. Am Fam Physician. (2015) 91:617-24.

36. Stein DJ, Scott KM, Jonge P de, Kessler RC. Epidemiology of anxiety disorders: from surveys to nosology and back. Dialogues Clin Neurosci. (2017) 19:127-36. doi: 10.31887/DCNS.2017.19.2/dstein

37. American Psychiatric Association. Diagnostic and Statistical Manual of Mental Disorders. Washington, DC: American Psychiatric Publishing (2013). doi: 10.1176/appi.books.9780890425596

38. Mansell W, Harvey A, Watkins ER, Shafran R. Cognitive behavioral processes across psychological disorders: a review of the utility and validity of the transdiagnostic approach. Int J Cogn Ther. (2008) 1:18191. doi: 10.1521/ijct.2008.1.3.181

39. Einstein DA. Extension of the transdiagnostic model to focus on intolerance of uncertainty: a review of the literature and implications for treatment. Clin Psychol Sci Pract. (2014) 21:280-300. doi: 10.1111/cpsp. 12077

40. Kessler RC. The national comorbidity survey of the United states. Int Rev Psychiatry. (1994) 6:365-76. doi: 10.3109/09540269409023274

41. Mansell W. The perceptual control model of psychopathology. Curr Opin Psychol. (2021) 41:15-20. doi: 10.1016/j.copsyc.2021.01.008

42. Harvey AG, Watkins E, Mansell W. Cognitive Behavioural Processes Across Psychological Disorders: A Transdiagnostic Approach to Research and Treatment. New York, NY: Oxford University Press. (2004) doi: 10.1093/med:psych/9780198528883.001.0001

43. Kim MJ, Loucks RA, Palmer AL, Brown AC, Solomon KM, Marchante $\mathrm{AN}$, et al. The structural and functional connectivity of the amygdala: from normal emotion to pathological anxiety. Behav Brain Res. (2011) 223:40310. doi: 10.1016/j.bbr.2011.04.025

44. Fiorillo CD, Tobler PN, Schultz W. Discrete coding of reward probability and uncertainty by dopamine neurons. Science. (2003) 299:1898-902. doi: 10.1126/science.1077349

45. Miyazaki K, Miyazaki KW, Yamanaka A, Tokuda T, Tanaka KF, Doya K. Reward probability and timing uncertainty alter the effect of dorsal raphe serotonin neurons on patience. Nat Commun. (2018) 9:2048. doi: 10.1038/s41467-018-04496-y
46. Herbert J. Testosterone, cortisol and financial risk-taking. Front Behav Neurosci. (2018) 12:101. doi: 10.3389/fnbeh.2018.00101

47. Slee A, Nazareth I, Bondaronek P, Liu Y, Cheng Z, Freemantle N. Pharmacological treatments for generalised anxiety disorder: a systematic review and network meta-analysis. Lancet. (2019) 393:768-77. doi: 10.1016/S0140-6736(18)31793-8

48. Jacobson GA, Diba K, Yaron-Jakoubovitch A, Oz Y, Koch C, Segev I, et al. Subthreshold voltage noise of rat neocortical pyramidal neurones. J Physiol. (2005) 564:145-60. doi: 10.1113/jphysiol.2004. 080903

49. McDonnell MD, Ward LM. The benefits of noise in neural systems: bridging theory and experiment. Nat Rev Neurosci. (2011) 12:41525. doi: $10.1038 / \mathrm{nrn} 3061$

50. Bach DR, Dolan RJ. Knowing how much you don't know: a neural organization of uncertainty estimates. Nat Rev Neurosci. (2012) 13:57286. doi: $10.1038 / \mathrm{nrn} 3289$

51. Anteraper SA, Triantafyllou C, Sawyer AT, Hofmann SG, Gabrieli JD, Whitfield-Gabrieli S. Hyper-connectivity of subcortical restingstate networks in social anxiety disorder. Brain Connect. (2014) 4:81-90. doi: 10.1089/brain.2013.0180

52. Van Beers RJ, Baraduc P, Wolpert DM. Role of uncertainty in sensorimotor control. Philos Trans $R$ Soc B Biol Sci. (2002) 357:1137-45. doi: 10.1098/rstb.2002.1101

53. Schultz W, Preuschoff K, Camerer C, Hsu M, Fiorillo CD, Tobler PN, et al. Review. explicit neural signals reflecting reward uncertainty. Philos Trans $R$ Soc B Biol Sci. (2008) 363:3801-11. doi: 10.1098/rstb.2008.0152

54. Huettel SA, Song AW, McCarthy G. Decisions under uncertainty: probabilistic context influences activation of prefrontal and parietal cortices. J Neurosci. (2005) 25:3304-11. doi: 10.1523/JNEUROSCI.5070-04.2005

55. Marshall L, Mathys C, Ruge D, de Berker AO, Dayan P, Stephan KE, et al. Pharmacological fingerprints of contextual uncertainty. PLoS Biol. (2016) 14:e1002575. doi: 10.1371/journal.pbio.1002575

56. Hardy B, Page L, Schaffner M, Graggaber J, Powlson AS, Fletcher PC, et al. Cortisol shifts financial risk preferences Narayanan Kandasamy1. Proc Natl Acad Sci USA. (2014) 111:3608-13. doi: 10.1073/pnas.1317908111

57. Gilbert K, Mineka S, Zinbarg RE, Craske MG, Adam EK. Emotion regulation regulates more than Emotion: associations of momentary emotion regulation with diurnal cortisol in current and past depression and anxiety. Clin Psychol Sci. (2017) 5:37-51. doi: 10.1177/2167702616654437

58. Hernández E, Lastra S, Urbina M, Carreira I, Lima L. Serotonin, 5hydroxyindoleacetic acid and serotonin transporter in blood peripheral lymphocytes of patients with generalized anxiety disorder. Int Immunopharmacol. (2002) 2:893-900. doi: 10.1016/S1567-5769(02)00025-5

59. Cervenka S, Hedman E, Ikoma Y, Djurfeldt DR, Rück C, Halldin C, et al. Changes in dopamine D2-receptor binding are associated to symptom reduction after psychotherapy in social anxiety disorder. Transl Psychiatry. (2012) 2:120. doi: 10.1038/tp.2012.40

60. Lesch KP, Bengel D, Heils A, Sabol SZ, Greenberg BD, Petri S, et al. Association of anxiety-related traits with a polymorphism in the serotonin transporter gene regulatory region. Science. (1996) 274:152731. doi: $10.1126 /$ science.274.5292.1527

61. Rosen NO, Ivanova E, Knäuper B. Differentiating intolerance of uncertainty from three related but distinct constructs. Anxiety Stress Coping. (2014) 27:55-73. doi: 10.1080/10615806.2013.815743

62. Haug TT, Mykletun A, Dahl AA. Are anxiety and depression related to gastrointestinal symptoms in the general population? Scand J Gastroenterol. (2002) 37:294-8. doi: 10.1080/003655202317284192

63. Mussell M, Kroenke K, Spitzer RL, Williams JBW, Herzog W, Löwe B. Gastrointestinal symptoms in primary care: prevalence and association with depression and anxiety. J Psychosom Res. (2008) 64:605-12. doi: 10.1016/j.jpsychores.2008.02.019

64. Jeon BH, Choi M, Lee J, Noh SH. Relationships between gastrointestinal symptoms, uncertainty, and perceived recovery in patients with gastric cancer after gastrectomy. Nurs Heal Sci. (2016) 18:23-9. doi: 10.1111/nhs.12219

65. Thayer JF, Friedman BH, Borkovec TD. Autonomic characteristics of generalized anxiety disorder and worry. Biol Psychiatry. (1996) 39:25566. doi: 10.1016/0006-3223(95)00136-0 
66. Ayling E, Aghajani M, Fouche JP, Van Der Wee N. Diffusion tensor imaging in anxiety disorders. Curr Psychiatry Rep. (2012) 14:197202. doi: $10.1007 / \mathrm{s} 11920-012-0273-\mathrm{z}$

67. Klumpp H, Fitzgerald JM. Neuroimaging predictors and mechanisms of treatment response in social anxiety disorder: an overview of the amygdala. Curr Psychiatry Rep. (2018) 20:1-9. doi: 10.1007/s11920-018-0948-1

68. Killgore WDS, Britton JC, Schwab ZJ, Price LM, Weiner MR, Gold AL, et al. Cortico-limbic responses to masked affective faces across PTSD, panic disorder, and specific phobia. Depress Anxiety. (2014) 31:1509. doi: $10.1002 /$ da. 22156

69. de Zambotti M, Trinder J, Silvani A, Colrain IM, Baker FC. Dynamic coupling between the central and autonomic nervous systems during sleep: a review. Neurosci Biobehav Rev. (2018) 90:84-103. doi: 10.1016/j.neubiorev.2018.03.027

70. Moreno-López Y, Olivares-Moreno R, Cordero-Erausquin M, Rojas-Piloni G. Sensorimotor Integration by Corticospinal System. Front Neuroanat. (2016) 10:24. doi: 10.3389/fnana.2016.00024

71. McNab F, Klingberg T. Prefrontal cortex and basal ganglia control access to working memory. Nat Neurosci. (2008) 11:103-7. doi: 10.1038/nn2024

72. Arsalidou M, Duerden EG, Taylor MJ. The centre of the brain: topographical model of motor, cognitive, affective, and somatosensory functions of the basal ganglia. Hum Brain Mapp. (2013) 34:3031-54. doi: 10.1002/hbm.22124

73. Wu JC, Buchsbaum MS, Hershey TG, Hazlett E, Sicotte N, Chad Johnson J. PET in generalized anxiety disorder. Biol Psychiatry. (1991) 29:118199. doi: 10.1016/0006-3223(91)90326-H

74. Sacchet MD, Prasad G, Foland-Ross LC, Joshi SH, Hamilton JP, Thompson PM, et al. Structural abnormality of the corticospinal tract in major depressive disorder. Biol Mood Anxiety Disord. (2014) 4:8. doi: 10.1186/2045-5380-4-8

75. Schutter DJLG, Hofman D, Van Honk J. Fearful faces selectively increase corticospinal motor tract excitability: a transcranial magnetic stimulation study. Psychophysiology. (2008) 45:3458. doi: 10.1111/j.1469-8986.2007.00635.x

76. Carrasco MA, Castro P, Sepulveda FJ, Tapia JC, Gatica K, Davis MI, et al. Regulation of glycinergic and GABAergic synaptogenesis by brain-derived neurotrophic factor in developing spinal neurons. Neuroscience. (2007) 145:484-94. doi: 10.1016/j.neuroscience.2006.12.019

77. Kaas JH. Somatosensory system. In: Mai JK, Paxinos G, editors. The Human Nervous System. London: Academic Press (2012). p. 1074109. doi: 10.1016/B978-0-12-374236-0.10030-6

78. Woll J, Sprenger A, Helmchen C. Postural control during galvanic vestibular stimulation in patients with persistent perceptual-postural dizziness. $J$ Neurol. (2019) 266:1236-49. doi: 10.1007/s00415-019-09255-7

79. Naranjo EN, Cleworth TW, Allum JHJJ, Inglis JT, Lea J, Westerberg BD, et al. Vestibulo-spinal and vestibulo-ocular reflexes are modulated when standing with increased postural threat. J Neurophysiol. (2015) 115:83342. doi: $10.1152 /$ jn. 00626.2015

80. Tanaka Y. Spinal reflexes during postural control under psychological pressure. Motor Control. (2015) 19:242-9. doi: 10.1123/mc.2013-0104

81. Davis JR, Horslen BC, Nishikawa K, Fukushima K, Chua R, Inglis JT, et al. Human proprioceptive adaptations during states of height-induced fear and anxiety. J Neurophysiol. (2011) 106:3082-90. doi: 10.1152/jn.01030.2010

82. Hoehn-Saric R, McLeod DR. The peripheral sympathetic nervous system. its role in normal and pathologic anxiety. Psychiatr Clin North Am. (1988) 11:375-86. doi: 10.1016/S0193-953X(18)30504-5

83. Maxwell JC. I. On governors. Proc R Soc London. (1868) 16:27083. doi: $10.1098 /$ rspl.1867.0055

84. Bennett S. A History of Control Engineering, 1930-1955? Peter Peregrinus (1993) doi: 10.1049/PBCE047E

85. Paraskevopoulos P. Modern Control Engineering?. Boca Raton: CRC Press (2017).

86. Craik KJW. Theory of the human operator in control systems: II. man as an element in a control system. Br J Psychol Gen Sect. (1948) 38:1428. doi: 10.1111/j.2044-8295.1948.tb01149.x

87. Wiener N. Cybernetics: Control and Communication in the Animal and the Machine? 2nd ed. Cambridge, MA: MIT Press (1961).

88. Ashby WR. Design for a Brain. 2nd ed. New York, NY: Wiley (1960).

89. Powers W. Behavior: The Control of Perception. Chicago, IL: Aldine (1973).
90. Mansell W, Marken RS. The origins and future of control theory in psychology. Rev Gen Psychol. (2015) 19:425-30. doi: 10.1037/gpr0000057

91. Vancouver JB. Self-regulation in organizational settings: a tale of two paradigms. In: Boekaerts M, Pintrich PR, Zeidner M, editors. Handbook of Self-Regulation. Cambridge MA: Academic Press (2000).303-41. doi: 10.1016/B978-012109890-2/50039-1

92. Vohs KD, Baumeister RF (editors.). Handbook of Self-Regulation: Research, Theory, and Applications. 2nd ed. New York, NY: Guilford Press (2011).

93. Cannon WB. The wisdom of the body. Am J Med Sci. (1932) 184:864. doi: 10.1097/00000441-193212000-00028

94. Powers WT. Making Sense of Behavior: The Meaning of Control. New Canaan, CT?: Benchmark (1998).

95. Gray JA. The Psychology of Fear and Stress -. London: McGraw-Hill (1971).

96. Carey TA. Exposure and reorganization: the what and how of effective psychotherapy. Clin Psychol Rev. (2011) 31:23648. doi: 10.1016/j.cpr.2010.04.004

97. Collins T, Tillmann B, Barrett FS, Delbé C, Janata P. A combined model of sensory and cognitive representations underlying tonal expectations in music: from audio signals to behavior. Psychol Rev. (2014) 121:3365. doi: $10.1037 / \mathrm{a} 0034695$

98. Vilares I, Howard JD, Fernandes HL, Gottfried JA, Kording KP. Differential representations of prior and likelihood uncertainty in the human brain. Curr Biol. (2012) 22:1641-8. doi: 10.1016/j.cub.2012.07.010

99. Toschi N, Duggento A, Passamonti L. Functional connectivity in amygdalar-sensory/(pre)motor networks at rest: new evidence from the Human Connectome Project. Eur J Neurosci. (2017) 45:12249. doi: 10.1111/ejn.13544

100. Jenks SK, Zhang S, Li C shan R, Hu S. Threat bias and resting state functional connectivity of the amygdala and bed nucleus stria terminalis. J Psychiatr Res. (2020) 122:54-63. doi: 10.1016/j.jpsychires.2019.12.017

101. Jahanshahi M, Obeso I, Rothwell JC, Obeso JA. A fronto-striatosubthalamic-pallidal network for goal-directed and habitual inhibition. Nat Rev Neurosci. (2015) 16:719-32. doi: 10.1038/nrn4038

102. Gunaydin LA, Kreitzer AC. Cortico-basal ganglia circuit function in psychiatric disease. Annu Rev Physiol. (2016) 78:327-50. doi: 10.1146/annurev-physiol-021115-105355

103. Hikosaka $\mathrm{O}$, Nakamura K, Nakahara $\mathrm{H}$. Basal ganglia orient eyes to reward. J Neurophysiol. (2006) 95:567-84. doi: 10.1152/jn.00458.2005

104. Panksepp J, Lane RD, Solms M, Smith R. Reconciling cognitive and affective neuroscience perspectives on the brain basis of emotional experience. Neurosci Biobehav Rev. (2017) 76:187215. doi: 10.1016/j.neubiorev.2016.09.010

105. Parvizi J, Damasio A. Consciousness and the brainstem. Cognition. (2001) 79:135-60. doi: 10.1016/S0010-0277(00)00127-X

106. Venkatraman A, Edlow BL, Immordino-Yang MH. The brainstem in emotion: a review. Front Neuroanat. (2017) 11:15. doi: $10.3389 /$ fnana.2017.00015

107. Sattin D, Leonardi M, Picozzi M. The autonomic nervous system and the brainstem: a fundamental role or the background actors for consciousness generation? Hypothesis, evidence, and future directions for rehabilitation and theoretical approaches. Brain Behav. (2020) 10:e01474. doi: 10.1002/brb3.1474

108. Chhetri PK, Das JM. Neuroanatomy, Neural Tube Development and Stages. Treasure Island (FL): StatPearls Publishing (2020).

109. Jorgensen EO. How the brain gets a roaring campfire: Input output functions. In: Mansell W, editor. The Interdisciplinary Handbook of Perceptual Control Theory: Living Control Systems IV. London: Academic Press (2020). p. e2.1-73. doi: 10.1016/B978-0-12-818948-1.00018-6

110. Jorgensen EO. How the brain gets a roaring campfire : structuring for perceptual results. In: Mansell W, editor. The Interdisciplinary Handbook of Perceptual Control Theory: Living Control Systems IV. London: Academic Press (2020). p. e1.1-1. doi: 10.1016/B978-0-12-818948-1.00017-4

111. Lang M, Krátký J, Shaver JH, Jerotijević D, Xygalatas D. Effects of anxiety on spontaneous ritualized behavior. Curr Biol. (2015) 25:18927. doi: 10.1016/j.cub.2015.05.049

112. Barlow DH. Unraveling the mysteries of anxiety and its disorders from the perspective of emotion theory. Am Psychol. (2000) 55:124763. doi: 10.1037/0003-066X.55.11.1247 
113. Zalsman G. Neurobiology of suicide in times of social isolation and loneliness. Eur Neuropsychopharmacol. (2020) 40:1-3. doi: 10.1016/j.euroneuro.2020.10.009

114. Pedersen ML, Frank MJ, Biele G. The drift diffusion model as the choice rule in reinforcement learning. Psychon Bull Rev. (2017) 24:123451. doi: 10.3758/s13423-016-1199-y

115. Ludvig EA, Sutton RS, Kehoe EJ. Stimulus representation and the timing of reward-prediction errors in models of the dopamine system. Neural Comput. (2008) 20:3034-54. doi: 10.1162/neco.2008.11-07-654

116. Powers W. Living Control Systems III: The Fact of Control?. Savannah, GA: Williams \& Company (2008).

117. Jorgensen EO. How the brain gets a roaring campfire: thalamus through a PCT microscope. In: Mansell W, editor. The Interdisciplinary Handbook of Perceptual Control Theory: Living Control Systems IV. Academic Press Inc. (1998). p. e3.1-42. doi: 10.1016/B978-0-12-818948-1. 00019-8

118. Eyre J, Miller S, Clowry G, Conway E, Watts W. Functional corticospinal projections are established prenatally in the human foetus permitting involvement in the development of spinal motor centres?. Brain? (2000) 121:51-64. doi: 10.1093/brain/123.1.51

119. Braga RM, Roze E, Ball G, Merchant N, Tusor N, Arichi T, et al. Development of the corticospinal and callosal tracts from extremely premature birth up to 2 years of age. PLoS ONE. (2015) 10:e125681. doi: 10.1371/journal.pone.0125681

120. Bowlby J. Attachment and Loss: Volume II: Separation, Anxiety and Anger?. New York, NY: Basic Books (1973).

121. Ainsworth M. Attachments other affectional bonds across the life cycle?. In: Parkes C, Stevenson-Hinde J, Marris P, editors. Attachment Across the Life Cycle. New York, NY: Routledge (1991). p. 33-51.

122. Mikulincer M, Shaver P. Attachment in Adulthood: Structure, Dynamics, and Change?. 2nd ed. New York, NY: Guilford Press (2016).

123. Gjerde PF, Onishi M, Carlson KS. Personality characteristics associated with romantic attachment: a comparison of interview and self-report methodologies. Personal Soc Psychol Bull. (2004) 30:1402-15. doi: 10.1177/0146167204264291

124. Mikulincer M. Adult attachment style and information processing: individual differences in curiosity and cognitive closure. $J$ Pers Soc Psychol. (1997) 72:1217-30. doi: 10.1037/0022-3514.72. 5.1217
125. Weber C, Federico CM. Interpersonal attachment and patterns of ideological belief. Polit Psychol. (2007) 28:389-416. doi: 10.1111/j.1467-9221.2007.00579.x

126. Gillath O, Karantzas G. Attachment security priming: a systematic review. Curr Opin Psychol. (2019) 25:86-95. doi: 10.1016/j.copsyc.2018.03.001

127. Nitschke JP, Forbes PAG, Ali N, Cutler J, Apps MAJ, Lockwood PL, et al. Resilience during uncertainty? Greater social connectedness during COVID19 lockdown is associated with reduced distress and fatigue. $\mathrm{Br} \mathrm{J}$ Health Psychol. (2020) 26:bjhp.12485. doi: 10.31234/osf.io/9ehm7

128. Giebel C, Cannon J, Hanna K, Butchard S, Eley R, Gaughan A, et al. Impact of COVID-19 related social support service closures on people with dementia and unpaid carers: a qualitative study. Aging Ment Health. (2020) 25:1-8. doi: 10.1080/13607863.2020.1822292

129. Nassar MR, McGuire JT, Ritz H, Kable JW. Dissociable forms of uncertaintydriven representational change across the human brain. J Neurosci. (2019) 39:1688-98. doi: 10.1101/364638

130. Smith BM, Twohy AJ, Smith GS. Psychological inflexibility and intolerance of uncertainty moderate the relationship between social isolation and mental health outcomes during COVID-19. J Context Behav Sci. (2020) 18:16274. doi: 10.1016/j.jcbs.2020.09.005

Conflict of Interest: The authors declare that the research was conducted in the absence of any commercial or financial relationships that could be construed as a potential conflict of interest.

Publisher's Note: All claims expressed in this article are solely those of the authors and do not necessarily represent those of their affiliated organizations, or those of the publisher, the editors and the reviewers. Any product that may be evaluated in this article, or claim that may be made by its manufacturer, is not guaranteed or endorsed by the publisher.

Copyright (C) 2021 Goldstein Ferber, Shoval, Zalsman, Mikulincer and Weller. This is an open-access article distributed under the terms of the Creative Commons Attribution License (CC BY). The use, distribution or reproduction in other forums is permitted, provided the original author(s) and the copyright owner(s) are credited and that the original publication in this journal is cited, in accordance with accepted academic practice. No use, distribution or reproduction is permitted which does not comply with these terms. 